\title{
Acometimento neonatal do pênfigo vulgar: relato de caso
}

Neonatal involvement of pemphigus vulgaris: clinical case

Pénfigo vulgar neonatal: reporte de caso

Mariana das Neves MELO'

Elisângela PEGAS ${ }^{2}$

${ }^{I}$ Residente de Dermatologia do Hospital PUC-Campinas, 13060-904 Campinas-SP, Brasil

${ }^{2}$ Médica Assistente do Ambulatório de Dermatologia da PUC-Campinas, 13060-904 Campinas-SP, Brasil

\section{INTRODUÇÃO}

Os pênfigos são dermatoses bolhosas autoimunes, em que há produção de autoanticorpos direcionados contra moléculas de adesão dos epitélios, levando à perda da coesão celular. As doenças bolhosas intraepidérmicas são compostas pelo grupo dos pênfigos: pênfigo foliáceo clássico, pênfigo foliáceo endêmico, pênfigo eritematoso, pênfigo vulgar, pênfigo vegetante, pênfigo herpetiforme (variante clínica dos pênfigos vulgar e foliáceo), pênfigo por IgA, pênfigo paraneoplásico e pênfigo induzido por medicamentos. Devido à transferência placentária dos anticorpos IgG, existem casos de recém-nascidos com diagnóstico de Pênfigo Neonatal.

\section{CASO CLÍNICO}

Paciente recém-nascida de parto normal em Hospital de Campinas-SP, do sexo feminino, idade gestacional de 37 semanas, peso $3325 \mathrm{~g}$, estatura 49 $\mathrm{cm}$. Apresentou ao nascimento bom estado geral, perfusão capilar preservada, eupneica, sem necessidade de assistência específica na sala de parto. À inspeção, foram visualizadas lesões exulceradas extensas na região perineal, couro cabeludo, cervical posterior e dorso, e também lesões bolhosas nos pés. Foram realizados exames gerais e sorologias, todos negativos. A mãe apresentava o diagnóstico de Pênfigo Vulgar há 3 anos, em acompanhamento no serviço de Dermatologia do mesmo hospital, e estava em uso de Prednisona, Metotrexato e Dapsona para controle da doença. A mesma realizou acompanhamento pré-natal no ambulatório de PréNatal de Alto Risco. A paciente se manteve em regime de internação em setor de UTI Neonatal para realização de curativos e cuidados diários. Não foi necessária introdução de medicação sistêmica específica. Foram realizados curativos com gaze impregnada e limpeza suave com compressas cirúrgicas, até a remissão do quadro (Figuras 1 a 3).

\section{DISCUSSÃO}

A gestação pode desencadear ou exacerbar o Pênfigo Vulgar. Com relação ao feto, pode ocorrer retardo de crescimento, prematuridade e morte intrauterina. Apesar de raro, há recém-nascidos que desenvolvem Pênfigo Neonatal. O quadro deve-se à passagem transplancentária de autoanticorpos IgG maternos e tende a regredir espontaneamente ao redor de três semanas, quando tais anticorpos são clareados pelo organismo do recém-nascido.
A manifestação clínica do pênfigo neonatal é menos grave em comparação à doença na mãe, não havendo acometimento sistêmico. No entanto, a gama de diagnósticos diferenciais é ainda mais ampla nos recém-nascidos do que nos adultos, pois a pele do neonato é particularmente propensa à formação de bolhas. Isso ocorre porque a pele fetal funcionalmente imatura inicia a queratinização nas semanas 22 a 24 de gestação e continua nas primeiras semanas após o nascimento.

Entre os casos de dermatoses bolhosas autoimunes em recém-nascidos, a maioria é de Pênfigo Vulgar, mas também foram descritos casos de Penfigoide Bolhoso, Lúpus Eritematoso Sistêmico Bolhoso, e Dermatose Bolhosa IgA Linear. Os locais de acometimento mais comum são dorso (63\%), cabeça e pescoço (60\%). Dentre os casos de pênfigo neonatal, $75 \%$ das mães apresentavam lesões de pele durante a gestação.
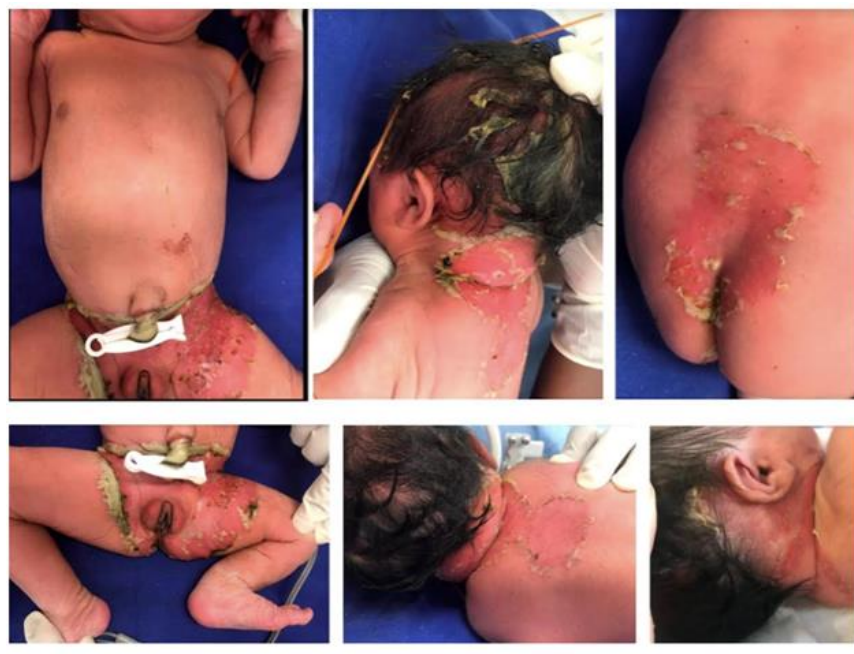

Figura 1: Recém-nascida com placas eritematosas exulceradas e crostas amarelo-hemáticas.
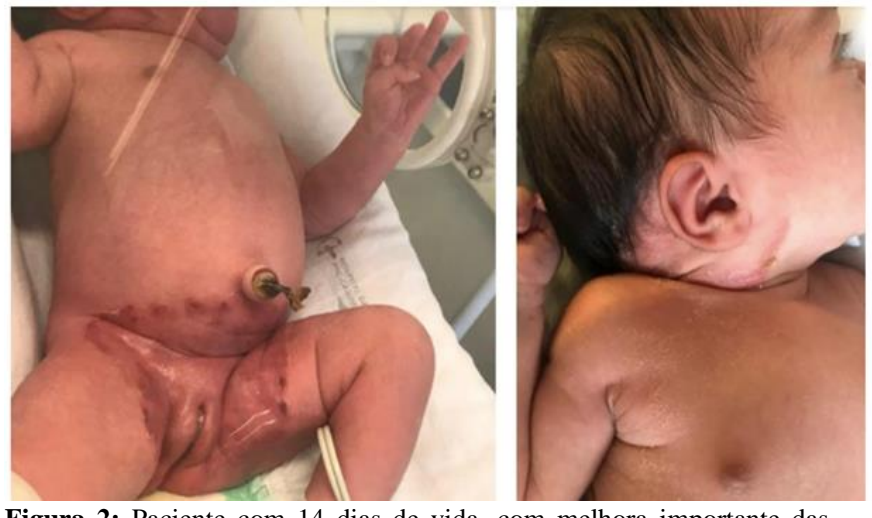

Figura 2: Paciente com 14 dias de vida, com melhora importante das lesões. 

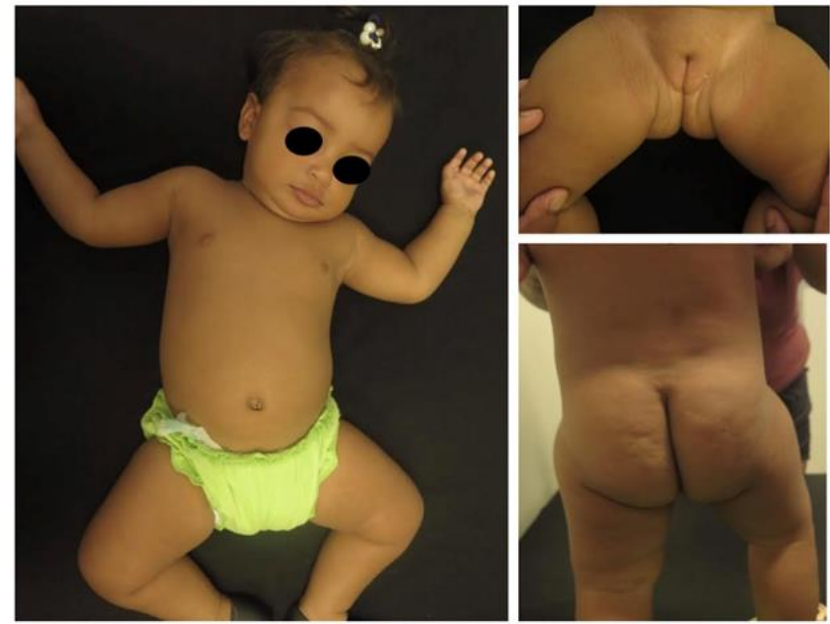

Figura 3: Paciente com 8 meses de idade e ausência de lesões.

CONCLUSÃO

O pênfigo neonatal pode apresentar manifestações como bolhas e erosões ao nascimento, alterações de mucosa, e até esfoliação extensa da pele. Algumas doenças devem ser excluídas, e o uso de antibióticos ou corticosteroides sistêmicos não é necessário. Os pacientes não apresentam probabilidade maior de doenças bolhosas na vida adulta e são assintomáticos antes do terceiro mês de vida. O diagnóstico do caso apresentado foi clínicoepidemiológico, após avaliação criteriosa das equipes de Dermatologia e Pediatria do serviço, e exclusão de outras enfermidades.

\section{REFERÊNCIAS}

1. Smolinski KN, Shah SS, Honig PJ, Yan AC. Neonatal cutaneous fungal infections. Curr Opin Pediatr. 2005;17(4):486-93.

2. Zhao CY, Chiang YZ, Murrell DF. Neonatal autoimmune blistering disease: a systematic review. Pediatr Dermatol. 2016;33(4):367-74.

3. Bellelli AG, Mantero NM, Rueda ML, Navacchia D, Cao G, Liloo L et al. Pénfigo vulgar de la infancia, a propósito de un caso [Childhood pemphigus vulgaris, a case report]. Arch Argent Pediatr. 2016;114(6):e457-60.

4. Ali HS. Pemphigus vulgaris during pregnancy - a case report. J Pak Assoc Dermatol. 2011; 21(4):301-3.

5. Hanna S, Kim M, Murrell DF. Validation studies of outcome measures in pemphigus. Int J Womens Dermatol. 2016;2(4):128-39.

6. Ibrahim SB, Yashodhara BM, Umakanth S, Kanagasabai S. Pemphigus vulgaris in a pregnant woman and her neonate. BMJ Case Rep. 2012;2012:bcr0220125850.

7. Kodagali SS, Subbarao SD, Hiremagaloor R. Pemphigus vulgaris in a neonate and his mother. Indian Pediatr. 2014;51(4):316-17.
8. Fainaru O, Mashiach R, Kupferminc M, Shenhav M, Pauzner D, Lessing JB. Pemphigus vulgaris in pregnancy: a case report and review of literature. Hum Reprod. 2000;15(5):1195-97.

9. Abrams ML, Smidt A, Benjamin L, Chen M, Woodley D, Mancini AJ. Congenital epidermolysis bullosa acquisita: vertical transfer of maternal autoantibody from mother to infant. Arch Dermatol. 2011;147(3):337-41.

10. Goldberg NS, DeFeo C, Kirshenbaum N. Pemphigus vulgaris and pregnancy: risk factors and recommendations. J Am Acad Dermatol. 1993;28(5 Pt 2):877-79.

\section{CONFLITO DE INTERESSES}

Os autores declaram não haver conflitos de interesse.

\section{AUTOR PARA CORRESPONDENCIA}

\section{Mariana das Neves Melo}

melo.marianan@gmail.com

Submetido em 01/06/2020

Aceito em 14/07/2020 